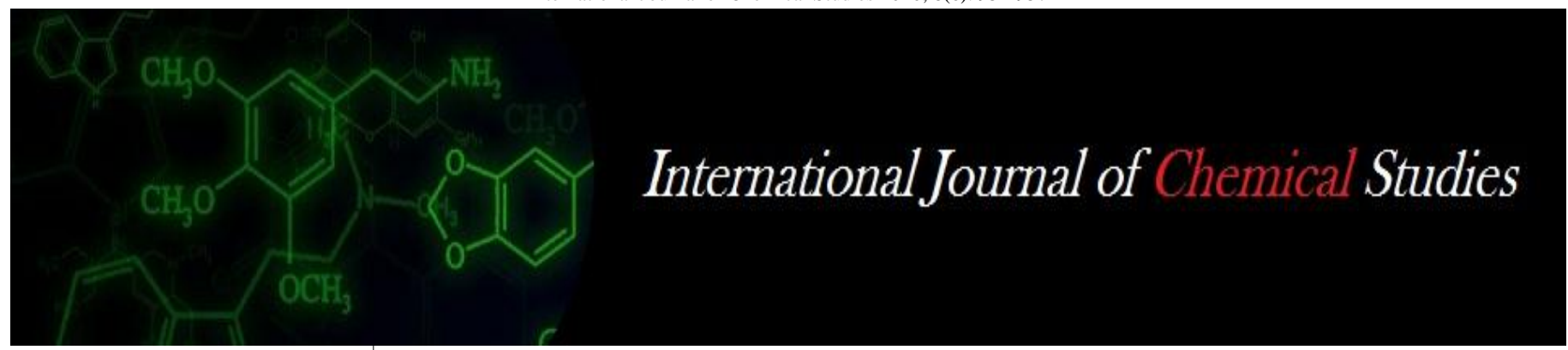

P-ISSN: 2349-8528

E-ISSN: 2321-4902

www.chemijournal.com

IJCS 2020; 8(6): 954-957

(C) 2020 IJCS

Received: 26-08-2020

Accepted: 12-10-2020

\section{HJ Bhalerao}

M.Sc. Scholar, Department of

Agril. Botany, Dr. P.D.K.V.,

Akola, Maharashtra, India

\section{Swati Bharad}

Senior Research Scientist

(Wheat), Wheat Research Unit,

Dr. P. D. K. V., Akola,

Maharashtra, India

\section{NR Potdukhe}

Senior Research Scientist

(Cotton), Cotton Research Unit,

Dr. P. D. K. V., Akola,

Maharashtra, India

SD Tayade

Senior Research Fellow, DUS Testing Project, S.T.R.U., Dr. P.D.K.V., Akola, Maharashtra, India

Corresponding Author: SD Tayade

Senior Research Fellow, DUS Testing Project, S.T.R.U., Dr. P.D.K.V., Akola, Maharashtra, India

\section{Combining ability for yield and it's contributing traits in wheat under heat stress condition (Triticum aestivum L.)}

\section{HJ Bhalerao, Swati Bharad, NR Potdukhe and SD Tayade}

DOI: https://doi.org/10.22271/chemi.2020.v8.i6n.10888

\begin{abstract}
Six lines and eight crosses were crossed in line $\mathrm{x}$ tester fashion to determine the general and specific combining ability variances for yield and yield contributing traits in wheat. The Analysis of Variance indicated that the differences due to crosses were significant for all the characters except protein percent. This indicated the presence of substantial genetic variability among the crosses for these characters. Among the parents two lines (AKAW-4627 and AKAW-4927) and two testers (C-306 and DHTW-60) are found to be good general combiners for yield per plant along with the most of the yield contributing traits. Thus, these parents should be included in further hybridization programme. The highest significant sca effect for grain yield was recorded by the cross AKAW-5023 x WH-7304 followed by AKAW-4627 x C-306, AKAW-4627 x DHTW-60, AKW-1071 x AKW-2862-1, NIAW-34 x AKAW-3717. The crosses AKW-1071 x HTW-11 and PDKV-Sardar x HINDI-62 recorded highly significant sca effects for protein percent and $\beta$-carotene content respectively. Among the genotypes AKAW-4627 x C-306, AKAW-4627 x DHTW-60 and AKAW-4927 x DHTW-60 are identified as best combiners for yield and yield contributing traits.
\end{abstract}

Keywords: General combining ability, Specific combing ability, ANOVA, hybrids, Triticum aestivum L.

\section{Introduction}

Wheat (Triticum aestivum L.) is one of the main cereal crop cultivated in respect of demands of population for food grain purpose. It is very important trade commodity as one fifth of the world wheat production is traded worldwide. It is known as king of cereal crop because its cultivation is easier and economically suitable. It is the most widely cultivated staple food in more than 40 countries for over $27 \%$ world's population. It is cultivated extensively in North Western and Central zones. New wheat cultivars are needed to adopt the crop to changing environment and meet the nutritional needs of the people particularly those in the developing countries. Hence, wheat breeders should start genetically enhancing the crop maintaining yield under high temperatures using all available means in the tool kit. Heat stress affects wheat plant senescence and photosynthesis, thereby influencing grain filling. Among various genetic techniques, combining ability analysis developed by Kempthorne (1957) ${ }^{[7]}$ provides important information for selection of parents in terms of the performance of their hybrids. Further it elucidates the nature and magnitude of various types of gene actions involved in the expression of quantitative characters. Combining ability has been defined and categorized originally by Spargue and Tatum (1942) who described that high general combining ability (GCA) effects were due to additive type of gene action, whereas high specific combining ability (SCA) indicated non-additive gene effects. Therefore the present studies conducted to assess the relative magnitude of GCA and SCA for yield and yield contributing traits and to select the best combiner for successful wheat hybridization.

\section{Materials and Methods}

The crosses were made using six lines (females) viz. NIAW-34, AKW-1071, PDKV-Sardar, AKAW-4627, AKAW-4927, AKAW-5023 and eight testers (males) viz., AKW-2862-1, C306, DHTW-60, WH-730, HTW-6, HINDI-62, AKAW-3717, HTW-11 and their 48 crosses along with the PDKV-Sardar as standard check have been evaluated in randomized block design with three replications at Wheat Research Unit, Dr. PDKV, Akola during rabi season of 2018-19. 
The resultant hybrids and parents were evaluated for days to heading, days to maturity, plant height $(\mathrm{cm})$ at maturity, number of tillers per plant at maturity, spike length, grains per spike, grain weight per spike, 1000 grain weight, grain yield per plant, protein percent $(\%), \beta$-carotene content (ppm).

\section{Results and Discussion}

The analysis of variance (line $\mathrm{x}$ tester) for eleven characters are given in Table 1 . The analysis of variance indicated that the differences due to crosses were significant for all the characters except protein percent. This indicated the presence of substantial genetic variability for these characters. Further, partitioning of genotypic variance into components viz., parents, parentsvs crosses and crosses revealed that the parents differed among themselves significantly for all the characters. The mean squares due to parents vs crosses differed significantly for all the characters. Similarly crosses also showed highly significant differences for all the traits except protein percent. The analysis of variance due to lines was significant for the all characters except viz., days to heading and protein percent. The analysis of variance due to testers was significant for all the characters except 1000 seed weight. The analysis of variance due to line $\mathrm{x}$ tester were significant for all characters except protein percent. Singh et al.(2007) ${ }^{[14]}$ and Kumar et al.(2018) ${ }^{[8]}$ also reported similar results. The knowledge of combining ability is a prerequisite to isolate the best specific combination and to study the combining ability of the parents with diverse genetic background. The gca effect is controlled by fixable additive genes and high gca would produce transgressive segregates in $\mathrm{F}_{2}$ or latter generation.

None of the parents recorded the significant gca in desirable direction simultaneously for all characters. However, AKW1071 and AKAW-4927 among the lines and C-306among the testers were found to possess significant gca effects for most of the yield contributing characters. Hence, these genotypes were observed to be good general combiners among the available genotypes. Whereas, among the genotypes NIAW34 was poor general combiners followed by AKAW-4627 and WH-730 for all traits. The estimates of gca revealed that among the lines, AKW-1071 was identified as good general combiner for number of tillers per plant, spike length and grain yield per plant.Line AKW-4927 was also identified as best combiner for spike length,number of seeds per spike, grain weight per spike, grain yield per plant (Table 2).

Among the six lines twoAKW-1071 and PDKV-Sardar possess negative (desirable) significant gca effect for days to heading. Lines parent AKW-5023 was also identified as best combiner having negative (desirable) significant gca effect for plant height. The Similar result on gca effects of various yield and yield contributing traits were also reported by Sharma et al. (2003) ${ }^{[13]}$, Chandrashekhar and Kerketta (2004) ${ }^{[3]}$, Dhanda et al. (2006) ${ }^{[4]}$, Hassan et al. (2007) ${ }^{[5]}$, Housmand and Vanda (2008), Akbar et al. (2009) [1], Padhar et al. (2010) [10], Kant et al. (2011), Barot et al. (2014) ${ }^{[2]}$, Shafiq et al. (2016) ${ }^{[12]}$, Murugan and Kannan (2017) ${ }^{[9]}$, Rajput and Kandalkar (2018) [11].

Among the testers parents, C-306 recorded significant gca effect for maximum characters like days to heading,plant height, number of tillers per plant, number of seeds per spike, grain weight per spike, 1000 seed weight and grain yield per plant. Testers parent DHTW-60 was also identified as best combiner for grain yield per plant and negative (desirable) significant gca effect for plant height. Among all the lines and testers parents only WH-730 possess significant gca effect forprotein content. On the basis overall gca effects of parents, four parents AKW-1071, AKAW-4927, C-306 and DHTW-60 were identified as best general combiner for seed yield and yield related traits.

Specific combining ability effects are indicative of heterosis. Similarly, they represent both dominant and epistatic gene actions (Sprague and Tatum, 1942) ${ }^{[15]}$. Specific combining ability is directly related to heterosis. The specific combining ability effects of promising cross combinations are presented in Table 3.

Among the $48 \mathrm{~F}_{1}$ 's seventeen crosses recorded positive significant sca effects for seed yield per plant. The hybrid AKAW-5023 x WH-730 (4.12) recorded highest and highly positive significant sca effectfollowed by AKAW-4627 x C306 (3.73), AKW-1071 x AKW-2862-1 (3.12), NIAW x AKAW-3717 (3.01) and AKAW-4627 x AKW-2862-1(2.99) for grain yield per plant. Though the five crosses visually AKAW-5023 x WH-730, AKAW-5023 x HTW-11, PDKVSardar x HTW-6, NIAW-34 x HTW-11 and PDKV-Sardar x AKW-2862-1 show positive significant sca effects for seed yield per plant, these crosses does not able to produce high positive standard heterosis may be due to the low $\mathrm{x}$ low gca effects of the parent involved in these five crosses. This indicates that only having high positive significant sca effects for seed yield does not give high mean seed yield per plant along with high standard heterosis. These crosses viz., AKAW-5023 x WH-730, AKAW-5023 x HTW-11, PDKVSardar x HTW-6, NIAW-34 x HTW-11 and PDKV-Sardar x AKW-2862-1 showed positive significant sca effects for seed yield per plant, but in these crosses high sca is not associated with positive heterosis so these three crosses will not be suitable for exploitation of heterois. Similar situation was observed by Jatav et al. (2014) ${ }^{[6]}$ and Murugan and Kannan (2017) ${ }^{[9]}$.

Table 1: Analysis of variance for combining ability in wheat

\begin{tabular}{|c|c|c|c|c|c|c|c|c|c|c|c|c|}
\hline \multirow[b]{2}{*}{$\begin{array}{c}\text { Sources of } \\
\text { Variation }\end{array}$} & \multirow[b]{2}{*}{ d.f. } & \multicolumn{11}{|c|}{ Mean sum of squares } \\
\hline & & $\begin{array}{l}\text { Days to } \\
\text { heading }\end{array}$ & $\begin{array}{c}\text { Days to } \\
\text { maturity }\end{array}$ & $\begin{array}{l}\text { Plant height } \\
\text { at maturity } \\
(\mathrm{cm})\end{array}$ & $\begin{array}{c}\text { Number of } \\
\text { tillers } \\
\text { (per plant) }\end{array}$ & \begin{tabular}{|c|} 
Spike \\
length \\
(cm)
\end{tabular} & $\begin{array}{c}\text { Number of } \\
\text { seed per } \\
\text { spike }\end{array}$ & \begin{tabular}{|c|} 
Grain \\
weight per \\
spike (g)
\end{tabular} & $\begin{array}{l}1000 \text { seed } \\
\text { weight }(\mathrm{g})\end{array}$ & $\begin{array}{c}\text { Grain yield } \\
\text { per plant } \\
\text { (g) }\end{array}$ & $\begin{array}{c}\text { Protein } \\
\text { percent } \\
(\%)\end{array}$ & $\begin{array}{c}\beta \text {-Carotene } \\
\text { content } \\
(\mathbf{p p m})\end{array}$ \\
\hline Replications & 2 & 8.22 & 12.19 & $86.20 *$ & $0.32 *$ & 0.07 & 3.96 & 001 & 1053 & 1.04 & 0.02 & 0.21 \\
\hline Females & 5 & $39 *$ & $57.33^{*}$ & .55 & 17.68 & $20.09 * *$ & 144.36 & 0.87 & 81.02 & 14.38 & 0.51 & 1.60 \\
\hline Males & 7 & $292.92 * *$ & $209.81 * *$ & 209.90 & $8.21 * *$ & 2.92 & $503.06^{*}$ & 0.78 & $376.04 *$ & 27.20 & 1.56 & 0.31 \\
\hline Females x Males & 35 & $23.60^{* *}$ & $23.01 * *$ & $185.88^{* *}$ & 8.48 & $1.95 * *$ & $217.82 * *$ & $0.59 * *$ & $126.12 * *$ & $23.28 * *$ & 0.75 & $0.67 * *$ \\
\hline Error & 94 & 5.08 & & & 0.2 & 0.23 & 11.41 & 0.07 & & 0.61 & 0.59 & 0.10 \\
\hline Total & 143 & 25.72 & 22.28 & 85.44 & 3.24 & 1.47 & 90.55 & 0.26 & 68.51 & 7.95 & 0.67 & 0.30 \\
\hline
\end{tabular}

Note: * Significant at $5 \%$ level of significance

** Significant at $1 \%$ level of significance 
Table 2: General combining ability effects of parents in wheat

\begin{tabular}{|c|c|c|c|c|c|c|c|c|c|c|c|c|}
\hline $\begin{array}{l}\text { Sr. } \\
\text { No }\end{array}$ & Parents & $\begin{array}{c}\text { Days to } \\
\text { heading }\end{array}$ & $\begin{array}{c}\text { Days to } \\
\text { maturity }\end{array}$ & \begin{tabular}{|c|} 
Plant \\
height at \\
maturity \\
$(\mathrm{cm})$
\end{tabular} & $\begin{array}{c}\text { Number of } \\
\text { tillers (per } \\
\text { plant) }\end{array}$ & $\begin{array}{c}\text { Spike } \\
\text { length } \\
(\mathrm{cm})\end{array}$ & $\begin{array}{c}\text { Number } \\
\text { of seed } \\
\text { per spike }\end{array}$ & \begin{tabular}{|c|} 
Grain \\
weight per \\
spike (g)
\end{tabular} & $\begin{array}{l}1000 \text { seed } \\
\text { weight }(\mathrm{g})\end{array}$ & $\begin{array}{c}\text { Grain } \\
\text { yield per } \\
\text { plant }(\mathrm{g})\end{array}$ & $\begin{array}{c}\text { Protein } \\
\text { percent } \\
(\%)\end{array}$ & $\begin{array}{c}\beta \text {-Carotene } \\
\text { content } \\
(\text { ppm })\end{array}$ \\
\hline & \multicolumn{12}{|c|}{ Lines (line) } \\
\hline 1 & NIAW-34 & $-0.88 *$ & -0.13 & 1.2 & -0.085 & 0.08 & 0.85 & $-0.16^{* *}$ & $-3.53 * *$ & -0.23 & -0.02 & -0.07 \\
\hline 2 & AKW-1071 & $-1.30 * *$ & -0.55 & -0.92 & $1.62 * *$ & $0.54 * *$ & $-1.80 * *$ & -0.012 & 1.64 & $0.38 * *$ & 0.2 & 0.1 \\
\hline 3 & PDKV-Sardar & $-1.88 * *$ & $2.52 * *$ & 0.15 & 0.11 & $0.24 * *$ & $1.84 * *$ & 0.067 & 0.49 & $-0.28 *$ & 0.11 & -0.12 \\
\hline 4 & AKAW-4627 & 0.81 & 0.15 & $-7.74 * *$ & $-0.30 * *$ & $-1.59 * *$ & 1.09 & 0.031 & 0.3 & $0.96 * *$ & -0.12 & $0.47 * *$ \\
\hline 5 & AKAW-4927 & $2.23 * *$ & 0.27 & $3.63 * *$ & $-0.73 * *$ & $1.07 * *$ & $2.12 * *$ & $0.30 * *$ & 1.19 & $0.44 * *$ & -0.18 & $-0.25 * *$ \\
\hline \multirow[t]{5}{*}{6} & AKAW-5023 & $1.02 *$ & $-2.26 * *$ & $3.67 * *$ & $-0.62 * *$ & $-0.35^{* * *}$ & $-4.11 * *$ & $-0.22 * *$ & -0.02 & $-1.26 * *$ & 0.018 & -0.11 \\
\hline & S.E. \pm & 0.43 & 0.49 & 0.89 & 0.09 & 0.09 & 0.61 & 0.05 & 0.94 & 0.14 & 0.16 & 0.06 \\
\hline & C.D. $\overline{5 \%}$ & 0.84 & 0.98 & 1.76 & 0.17 & 0.18 & 1.21 & 0.1 & 1.86 & 0.29 & 0.32 & 0.13 \\
\hline & C.D. $1 \%$ & 1.12 & 1.3 & 2.33 & 0.23 & 0.23 & 1.61 & 0.13 & 2.47 & 0.38 & 0.42 & 0.17 \\
\hline & \multicolumn{12}{|c|}{ Male (Testers) } \\
\hline 7 & AKW-2862-1 & $-1.83 * *$ & -0.65 & $-3.37 * *$ & $-0.54 * *$ & 0.06 & $-5.14 * *$ & $0.19 * *$ & $6.50 * *$ & 0.158 & 0.29 & 0.02 \\
\hline 8 & C-306 & $-1.05^{*}$ & 1.01 & $-3.17 * *$ & $0.63 * *$ & 0.12 & $3.61 * *$ & $0.39 * *$ & $4.02 * *$ & $1.41 * *$ & $-0.44 *$ & $-0.16^{*}$ \\
\hline 9 & DHTW-60 & $-7.27 * *$ & $-6.81 * *$ & $3.48 * *$ & $-0.49 * *$ & $-0.20 *$ & $-3.96 * *$ & -0.07 & 1.58 & $1.43 * *$ & -0.07 & -0.05 \\
\hline 10 & WH-730 & 0.38 & 0.34 & -0.9 & $0.43 * *$ & $0.42 * *$ & $-3.24 * *$ & -0.03 & -1.14 & -0.25 & $0.38 *$ & $0.28 * *$ \\
\hline 11 & HTW-6 & $1.0^{*}$ & -0.43 & -1.94 & $0.83 * *$ & 0.01 & $7.77 * *$ & -0.09 & $-5.86^{* * *}$ & $-0.49 * *$ & -0.27 & -0.01 \\
\hline 12 & HINDI-62 & $7.33 * *$ & $5.73 * *$ & -1.51 & $-0.87 * *$ & $-0.88^{* * *}$ & $-3.78 * *$ & $-0.30 * *$ & -1.86 & $-2.41 * *$ & -0.12 & -0.04 \\
\hline 13 & AKAW-3717 & 0.22 & 0.18 & $6.31 * *$ & $0.56^{* *}$ & $0.23 *$ & $7.03 * *$ & -0.07 & $-6.13 * *$ & $0.50 * *$ & 0.3 & -0.05 \\
\hline \multirow[t]{4}{*}{14} & HTW-11 & $1.22 *$ & 0.62 & 1.12 & $-0.55 * *$ & $0.23 *$ & $-2.32 * *$ & 0.001 & $2.90 * *$ & $-0.34 *$ & -0.04 & 0.03 \\
\hline & S.E. \pm & 0.49 & 0.57 & 1.02 & 0.1 & 0.1 & 0.71 & 0.06 & 1.08 & 0.17 & 0.18 & 0.07 \\
\hline & C.D. 5\% & 0.97 & 1.13 & 2.03 & 0.2 & 0.2 & 1.4 & 0.11 & 2.15 & 0.33 & 0.37 & 0.15 \\
\hline & C.D. $1 \%$ & 1.29 & 1.5 & 2.69 & 0.27 & 0.27 & 1.86 & 0.15 & 2.85 & 0.44 & 0.49 & 0.19 \\
\hline
\end{tabular}

Note: *Significant at $5 \%$ level of significance

** Significant at $1 \%$ level of significance

Table 3: Estimation of Specific combining ability effects in wheat

\begin{tabular}{|c|c|c|c|c|c|c|c|c|c|c|c|c|}
\hline $\begin{array}{l}\text { Sr. } \\
\text { No }\end{array}$ & Crosses & $\begin{array}{c}\text { Days } \\
\text { to } \\
\text { heading }\end{array}$ & $\begin{array}{c}\text { Days } \\
\text { to } \\
\text { maturity }\end{array}$ & \begin{tabular}{|c|} 
Plant \\
height at \\
maturity \\
$(\mathrm{cm})$
\end{tabular} & $\begin{array}{c}\text { Number } \\
\text { of tillers } \\
\text { (per plant) }\end{array}$ & \begin{tabular}{|c|} 
Spike \\
length \\
$(\mathrm{cm})$
\end{tabular} & $\begin{array}{c}\text { Number of } \\
\text { Seeds per } \\
\text { Spike }\end{array}$ & $\begin{array}{c}\text { Grain } \\
\text { weight } \\
\text { per spike } \\
\text { (g) }\end{array}$ & $\begin{array}{c}1000 \\
\text { seed } \\
\text { weight }(g)\end{array}$ & $\begin{array}{c}\text { Grain } \\
\text { yield per } \\
\text { plant (g) }\end{array}$ & $\begin{array}{c}\text { Protein } \\
\text { percent } \\
(\%)\end{array}$ & $\begin{array}{c}\beta \text {-Carotene } \\
\text { content } \\
(\text { ppm) }\end{array}$ \\
\hline 1 & NIAW-34 X AKW-2862-1 & $-2.50 *$ & -1.30 & $-8.30 * *$ & $-0.79 * *$ & -0.25 & $-5.19^{* *}$ & $0.71 * *$ & $5.59^{*}$ & $-3.20 * *$ & -0.65 & $-0.64 * *$ \\
\hline 2 & NIAW-34 X C-306 & 1.72 & 2.36 & $22.26 * *$ & $0.73^{* * *}$ & $-0.51 *$ & $-11.24 * *$ & $-0.61 * *$ & -0.68 & $-2.89 * *$ & -0.40 & $0.37 *$ \\
\hline 3 & NIAW-34 X DHTW-60 & 2.27 & -2.1 & 2.98 & $-2.24 * *$ & 0.30 & $-10.80 * *$ & $-0.51 * *$ & -0.55 & $1.44 * *$ & 0.24 & $-0.38^{*}$ \\
\hline 4 & NIAW-34 X WH-730 & $-2.38^{*}$ & -1.63 & 0.50 & 0.26 & 0.05 & $-9.72 *$ & -0.14 & $10.62 * *$ & -0.79 & 0.25 & $0.48 * *$ \\
\hline 5 & NIAW-34X HTW-6 & 0.33 & -0.19 & $-6.26^{*}$ & $0.86^{* * *}$ & 0.16 & $14.06^{* * *}$ & 0.02 & $-7.41 * *$ & -0.28 & 05 & $0.37 *$ \\
\hline 6 & NIAW-34 X HINDI-62 & $2.6^{*}$ & 1.30 & -1.82 & $0.87 * *$ & 0.05 & $12.89 * *$ & 0.12 & $-7.02 * *$ & 0.78 & 29 & 0.34 \\
\hline 7 & NIAW-34X AKAW-3717 & 0.11 & -0.47 & 3.13 & $2.00 * *$ & $0.88 * *$ & $14.81 * *$ & $0.68 * *$ & 2.08 & $3.01 * *$ & .08 & .00 \\
\hline 8 & NIAW-34X HTW-11 & -2.22 & 2.08 & $-12.49 * *$ & 0.01 & -0.36 & $-4.79 * *$ & $-0.28 *$ & -2.61 & $1.94 * *$ & .30 & 0.14 \\
\hline 9 & AKW-1071X AKW-2862-1 & -0.41 & -1.22 & 0.66 & $1.86^{* * *}$ & -0.14 & 2.44 & $-0.38 * *$ & $-7.46^{*}$ & $3.12 * *$ & .63 & $0.56^{* * *}$ \\
\hline 10 & AKW-1071 X C-306 & $2.47 *$ & 1.11 & 0.73 & 0.25 & 0.16 & 14.99 ** & $0.90 * *$ & 1.69 & $-1.59 * *$ & 0.16 & 0.04 \\
\hline 11 & AKW-1071 X DHTW-60 & -1.97 & -2.05 & -0.44 & $-1.6^{* *}$ & -0.12 & -0.73 & $-0.33^{*}$ & $-5.47 *$ & $-4.20 * *$ & -0.33 & -0.12 \\
\hline 12 & $\mathrm{H}-730$ & 30 & -2.22 & $-5.38 *$ & $-0.64 *$ & $-0.97 * *$ & $4 * *$ & -0.07 & -5.17 & *** & $-0.93 *$ & 15 \\
\hline 13 & $\mathrm{~N}-6$ & 75 & 1.22 & 0.40 & -0.24 & $0.93 * *$ & $-12.34 * *$ & 0.20 & $12.75^{* *}$ & $2.19^{* *}$ & -0.68 & 4 \\
\hline 14 & $\overline{\mathrm{AK}}$ & $58 *$ & 0.72 & $5.30^{*}$ & * & $1.26 * *$ & & 0.06 & 3.44 & $-0.93 *$ & 46 & 9* \\
\hline 15 & AKW-1071X AKAW-3717 & $2.52 *$ & $2.94 *$ & $-7.69 * *$ & 0.29 & $-1.14^{* * *}$ & $-6.60 * *$ & $-0.36^{*}$ & -2.67 & 0.76 & 0.56 & 0.33 \\
\hline 16 & AKW-1071 X HTW-11 & 0.52 & -0.50 & $6.40^{*}$ & $-0.99 * *$ & 0.03 & -3.42 & -0.01 & 2.89 & 1.87 ** & $1.05^{*}$ & $-0.44 *$ \\
\hline 17 & PDKV-Sardar X AKW-2862-1 & $5.16^{* *}$ & -2.63 & 4.10 & -0.29 & $-0.52 *$ & -3.00 & -0.21 & 0.42 & $1.57 * *$ & 0.60 & -0.20 \\
\hline 18 & $\mathrm{YC} 306$ & $2.72 *$ & $-6.63 * *$ & -1.77 & F* & $-0.85^{* * *}$ & & -0.14 & -0.75 & $-2.66 * *$ & .42 & $-0.55 * *$ \\
\hline 19 & PDKV-Sar & 1.61 & 1.52 & $-5.26^{*}$ & $3.32 * *$ & 0.06 & $-7.28 * *$ & $-0.41 * *$ & -1.46 & $1.25^{* *}$ & .58 & $-0.41 *$ \\
\hline 20 & & $-2.72 *$ & $4.64 * *$ & $-9.97 * *$ & & $-1.13^{* *}$ & $7.54 * *$ & $-0.51 * *$ & $-11.38 * *$ & $-1.84 * *$ & -0.22 & $-0.65 * *$ \\
\hline 21 & HIW- & -2.33 & $-4.19 * *$ & $5.95^{*}$ & $0.90^{* * *}$ & 0.41 & $4.07 *$ & 0.07 & -3.05 & $2.19^{* *}$ & .06 & -0.00 \\
\hline 22 & PDKV-Sardar X HINDI-62 & $-2.66 *$ & $3.63^{*}$ & 4.28 & $-2.06 * *$ & $1.04 * *$ & 2.58 & 0.19 & 0.16 & $0.82^{*}$ & -0.20 & $0.74 * *$ \\
\hline 23 & PDKV-Sardar X AKAW-3717 & -0.88 & 2.52 & 3.53 & $-1.99 * *$ & -0.32 & $-5.38 * *$ & $0.44 * *$ & $10.93 * *$ & -0.14 & 0.15 & $0.53 * *$ \\
\hline 24 & PDKV-SardarX HTW-11 & -0.88 & 1.08 & -0.86 & $-1.98 * *$ & $1.32 * *$ & $4.47 *$ & $0.57 * *$ & 5.13 & $-1.19 * *$ & -0.10 & $0.54 * *$ \\
\hline 25 & KAW-4627 X & 0 & -0.26 & 2.58 & $2^{* *}$ & 0.25 & & 0.22 & 4.64 & $2.99^{* *}$ & .01 & 0.31 \\
\hline 26 & 7041607 & $-3.98 * *$ & 0.06 & $-5.50 *$ & 0.014 & $1.32 * *$ & 6 & $0.39 * *$ & $7.74 * *$ & $3.73^{* * *}$ & 6 & $02+2$ \\
\hline 27 & 6 & & 2.56 & -4.80 & *** & -0.21 & $5 * *$ & $0.38 * *$ & -0.34 & $3.54 * *$ & .37 & $0.64 * * *$ \\
\hline 28 & AW-4627 X WH-730 & 43 & 2.73 & $9.52 * *$ & $0.52 *$ & 0.48 & 3.18 & 0.01 & 0.32 & $2.29 * *$ & 0.46 & 0.11 \\
\hline 29 & AKAW-4627X HTW-6 & -1.37 & -0.48 & 1.64 & -0.44 & $-0.90 * *$ & 0.42 & 0.14 & 0.10 & $-3.99 * *$ & 0.39 & $-1.09 * *$ \\
\hline 30 & AKAW-4627 X HINDI-62 & 0.62 & -1.65 & $-7.83 * *$ & $-1.60 * *$ & -0.47 & $-9.67 * *$ & $-0.45 * *$ & -2.19 & $-0.99 *$ & 0.39 & 0.02 \\
\hline 31 & AKAW-4627 X AKAW-3717 & -0.26 & -2.76 & -1.87 & $-1.07 * *$ & 0.03 & -0.78 & $-0.67 * *$ & $-11.39 * *$ & $-4.26 * *$ & -0.18 & -0.24 \\
\hline 32 & AKAW-4627 X HTW-11 & $5.40 * *$ & -0.20 & $6.26^{*}$ & $0.83^{* * *}$ & $-0.49^{*}$ & -2.08 & -0.02 & 1.11 & $-3.31 * *$ & -0.21 & -0.02 \\
\hline 33 & AKAW-4927 X A & 1.04 & $3.94 * *$ & 3.58 & -0.04 & & -2.53 & -0.27 & 2.81 & -0.19 & 0.06 & 0.09 \\
\hline 34 & AKAW-4927 X C-306 & .06 & 0.27 & $-19.22 * *$ & $-2.18 * *$ & $0.80 * *$ & -2.33 & $-0.32^{*}$ & -1.18 & 0.42 & .05 & 0.02 \\
\hline 35 & A & & & $9.2^{* * *}$ & & 0.28 & & & $8.65 * *$ & $2.29 * *$ & 0.55 & $-0.37 *$ \\
\hline 36 & AW-4927 X WH-730 & $.81 * *$ & -2.05 & 1.23 & $-0.75 * *$ & 0.37 & $-10.49 * *$ & $0.67 * *$ & 2.28 & $-2.57 * *$ & 0.10 & -0.28 \\
\hline 37 & AKAW-4927X HTW-6 & -1.79 & 1.72 & $-6.93 * *$ & -0.31 & 0.01 & $4.82 * *$ & $-0.41 * *$ & $-8.17 * *$ & $0.86^{*}$ & 0.20 & $0.46^{*}$ \\
\hline 38 & AKAW-4927 X HINDI-62 & 0.87 & 0.88 & $8.31^{* *}$ & $2.28 * *$ & $-0.60 *$ & -1.52 & -0.00 & 3.91 & 0.14 & -0.45 & $-0.40 *$ \\
\hline
\end{tabular}




\begin{tabular}{|c|c|c|c|c|c|c|c|c|c|c|c|c|}
\hline 39 & AKAW-4927 X AKAW-3717 & -0.34 & $-3.88 * *$ & 4.95 & $1.55^{* * *}$ & 0.05 & $-7.10 * *$ & $-0.37 * *$ & 1.08 & $0.83^{*}$ & -0.17 & $0.36^{*}$ \\
\hline 40 & AKAW-4927 X HTW-11 & $-4.34 * *$ & -1.66 & -1.20 & $-1.73 * *$ & $-1.20 * *$ & $12.39 * *$ & -0.09 & $-9.40 * *$ & $-1.79 * *$ & -0.35 & 0.11 \\
\hline 41 & AKAW-5023 X AKW-2862-1 & $-3.08 *$ & 1.48 & -2.63 & $-1.65 * *$ & 0.40 & $6.75 * *$ & -0.06 & $-6.012 *$ & $-4.28 * *$ & -0.62 & -0.12 \\
\hline 42 & AKAW-5023X C-306 & -1.86 & $2.81 *$ & 3.50 & $-1.02 * *$ & $-0.92 * *$ & 3.15 & -0.21 & $-6.81 *$ & $2.99 * *$ & 0.22 & -0.15 \\
\hline 43 & AKAW-5023 X DHTW-60 & -0.97 & -0.68 & -1.74 & $-1.60 * *$ & -0.31 & 3.07 & 0.07 & -0.81 & $-4.33 * *$ & 0.49 & $0.64 * *$ \\
\hline 44 & AKAW-5023 X WH-730 & 0.02 & -1.51 & 4.09 & $0.87 * *$ & $1.20 * *$ & 0.95 & 0.04 & 3.32 & $4.12 * *$ & 0.34 & $0.49 * *$ \\
\hline 45 & AKAW-5023X HTW-6 & $4.41 * *$ & 1.93 & $5.20^{*}$ & $0.97 * *$ & -0.29 & $-11.03 * *$ & -0.03 & $5.77 *$ & $-0.96^{*}$ & 0.09 & 0.20 \\
\hline 46 & AKAW-5023X HINDI-62 & 1.08 & $-4.90 * *$ & $-8.24 * *$ & $-0.62 *$ & $-1.28 * *$ & -1.40 & 0.07 & 1.69 & 0.18 & 0.43 & $-0.41 *$ \\
\hline 47 & AKAW-5023X AKAW-3717 & -1.13 & 1.65 & -2.064 & $-0.78 * *$ & $0.50 *$ & $5.07 * *$ & $0.27 *$ & -0.02 & -0.20 & -0.28 & -0.31 \\
\hline 48 & AKAW-5023X HTW-11 & 1.52 & -0.79 & 1.897 & $3.85 * *$ & 0.71 ** & $-6.56^{* * *}$ & -0.16 & 2.87 & $2.48 * *$ & -0.68 & -0.33 \\
\hline & $\mathrm{SE}(\mathrm{D}) \pm$ & 1.20 & 1.40 & 2.51 & 0.25 & 0.25 & 1.73 & 0.14 & 2.65 & 0.41 & 0.45 & 0.18 \\
\hline & $\mathrm{CD}(5 \%)$ & 2.39 & 2.78 & 4.97 & 0.49 & 0.50 & 3.43 & 0.28 & 5.27 & 0.81 & 0.90 & 0.36 \\
\hline & $\mathrm{CD}(1 \%)$ & 3.16 & 3.68 & 6.59 & 0.65 & 0.66 & 4.55 & 0.37 & 6.97 & 1.07 & 1.19 & 0.47 \\
\hline
\end{tabular}

Note: $*$ Significant at $5 \%$ level of significance

** Significant at $1 \%$ level of significance

\section{Conclusion}

The extent GCA was higher than SCA for all the characters indicates towards existence of genetic variability in the parental lines included in the present study and involvement of both additive and non-additive gene effects in the inheritance of these traits. In case of the specific combining ability, none of the cross showed the significant sca effects in desirable direction simultaneously for all the characters studied. The highest significant sca effect for grain yield was recorded by the cross AKAW-5023 x WH-7304 followed by AKAW-4627 x C-306, AKAW-4627 x DHTW-60, AKW1071 x AKW-2862-1, NIAW-34 x AKAW-3717. The crosses AKW-1071 x HTW-11 and PDKV-Sardar x HINDI-62 recorded highly significant sca effects for protein percent and $\beta$-carotene content respectively. However, the crosses AKW1071 x C-306 and AKW-1071 x C-306 recorded highly significant sca effects for, grains per spike and grain weight per spike respectively. None of the parents showed the significant gca effects in desirable direction simultaneously for all the characters studied. However, among the parents, lines AKAW-4627, AKW-1071, AKAW-4927 and testers C306, DHTW-60, AKW-2862-1 and AKAW-3717 were identified as best general combiner for seed yield and yield contributing traits. Tester WH-730 was identified as the good general combiner for protein percent and $\beta$-carotene content. Whereas, in parents two lines AKAW-4627 and AKAW-4927 and two testers C-306 and DHTW-60 were good general combiner for the days to heading, plant height, number of tillers per plant, spike length, grains per spike, grain weight per spike, 1000 grain weight and grain yield per plant. Hence, these parents should be exploited in hybridization program to check grain yield under the heat stress condition.

\section{References}

1. Akbar M, Javed A, Mukhdoom H, Mujahid HQ, Khan S. Line $\times$ tester analysis in bread wheat (Triticum aestivum l.) J Agric. Res 2009;47(1):411-420.

2. Barot HG, Patel MS, Sheikh WA, Patel LP, Allam CR. Heterosis and combining ability analysis for yield and its component traits in wheat (Triticum aestivum L.). Electr. J. Plant Breed 2014;5(3):350-359.

3. Chandrashekhar M, Kerketta V. Estimation of some genetic parameters components in bread wheat. Int. J. Biol. Sci 2004;4(2):214-216.

4. Dhanda BA, Dobariya KL. Combining ability analysis over environment for grain yield and its components in bread wheat (T.aestivum L.). Natl. J. Pl. Improv 2006;8(2):172-173.

5. Hassan G, Mohammad F, Afridi SS, Khalil I. Combining ability in the F1 generations of diallel cross for yield and yield components in wheat. Sarhad J. Agric 2007;23(4):937-942.

6. Jatav KS, Agrwal RM, Tomar NS, Tyagi SR. Nitrogen metabolism, growth and yield responses of wheat (Triticum aestivum L.) to restricted water supply and varying potassium treatments. J. Indian bot. Soc 2014;93(3):177-189.

7. Kempthorne O. An introduction to genetic statistics, John Wiley and Son Inc. Newyork, Champman and Hall Ltd. London 1957, pp. 468-470.

8. Kumar J, Singh SK, Singh L, Kumar M, Srivastava M, Singh J, et al. Combining ability analysis for yield and its components in bread wheat (Triticum aestivum L.). Int. J. Curr. Microbial. App. Sci 2018;6(3):24-39.

9. Murugan A, Kannan R. Heterosis and combining ability analysis for yield trait of indian hexaploid wheat (Triticum aestivum) International Journal of Recent Scientific Research 2017;8:18242-18246.

10. Padhar PR, Madaria RB, Vachhani JH, Dobariya KL. Combining ability analysis of grain yield and its contributing characters in bread wheat (Triticum aestivum L.) under late sown condition. IJASc 2010;6:267-272.

11. Rajput RS, Kandalkar VS. Combining ability and heterosis for grain yield and its attributing traits in bread wheat (Triticum aestivum L) Journal of Pharmacognosy and Phytochemistry 2018;7(2):113-119

12. Shafiq AA, Abdul WB, Munaiza B, Ghulam AB, Tariq $\mathrm{AB}$, Aijaz AS, et al. Line $\times$ tester analysis for estimating combining ability in F1 hybrids of bread wheat Pure Appl. Biol 2016;5(3):647-652

13. Sharma S, Sohu VS, Mavi GS. Evaluation of elite wheat genotypes to quantitative characters in bread wheat. Asian J. Pl. Sci. 2003;3(4):508-511.

14. Singh J, Garg DK, Raje RS. Combining ability and gene action for grain yield and its components under high temperature environment in bread wheat (Triticum aestivum L). Indian J. Genet. 2007;67:193-195.

15. Sprague GF, LA Tatum. General Vs. specific combining ability in a single cross corn. J Amer. Soc. Agron 1942;34:923-932.

16. Subhani GM, Chowdhary MA. Genetic studies in bread wheat under irrigated and drought stress conditions. Pak. J. Biol. Sci 2000;3:1793-1798. 